\title{
SURGERY FOR THORACIC ESOPHAGEAL CARCINOMA WITH CLINICALLY POSITIVE CERVICAL NODES
}

Yuji Tachimori, MD

Hoichi Kato, MD

Hiroshi Watanabe, MD
Objective: We attempted to determine whether and under what circumstances surgical intervention should be recommended for patients with clinically positive cervical nodes for metastasis from thoracic esophageal carcinoma by pretreatment examination. Methods: The survival of the patients was compared using factors including anatomic subsites of the tumor, $\mathbf{T}$ categories, subdivisions of $\mathbf{N}$ categories, and $\mathbf{R}$ classification according to the TNM classification. Results: The 5-year survival of 63 patients who underwent surgery for primary therapy was $26.7 \%$. There was no statistically significant difference between the postoperative survivals when compared according to the location of the tumors. As a matter of course, the patients with later stages of the disease had worse survival. However, by the multivariable analysis, significant difference was recognized between the patients with tumor invading adjacent structures (T4) and those without and between the patients who received curative resection and those who did not. The 5-year survival of 47 patients who underwent surgery without residual tumor was $32.9 \%$. Conclusion: Even when the patient had clinically positive cervical nodes from thoracic esophageal carcinoma, they had a possibility of long-term survival after curative resection with neck lymph node dissection. An operation is thus indicated for curative intent. Accurate pretreatment evaluation is important when deciding which patients require surgery and how to carry out a curative surgical procedure without residual tumor. (J Thorac Cardiovasc Surg 1998;116:954-9)
$\mathrm{D}$ espite recent advances in the treatment of esophageal cancer, the general prognosis for patients with this disease remains poor. Most affected patients are seen at a late stage of the disease. The high incidence of cervical lymph node involvement in patients with thoracic esophageal carcinoma is well known. ${ }^{1}$ Altorki and Skinner ${ }^{2}$ recently reported the $35 \%$ prevalence of cervical node metastases in patients who undergo en bloc or standard esophagectomy. According to the TNM classification, ${ }^{3}$ metastatic involvement of

From the Department of Surgery, National Cancer Center Hospital, Tokyo, Japan.

This study was funded from Foundation for Promotion of Cancer Research and in part by Grants-in-Aid for Cancer Research (Nos. 5S-1 and 5-12) from the Ministry of Health and Welfare, Japan.

Received for publication Sept 5, 1997; revisions requested Feb 9, 1998; revisions received Aug 20, 1998; accepted for publication Aug 28, 1998.

Address for reprints: Y. Tachimori, MD, Department of Surgery, National Cancer Center Hospital, 1-1 Tsukiji 5-Chome, Chuoku, Tokyo 104, Japan.

Copyright () 1998 by Mosby, Inc.

0022-5223/98 $\$ 5.00+0 \quad \mathbf{1 2 / 1 / 9 4 2 2 8}$ the cervical nodes in patients with thoracic esophageal carcinoma is associated with distant metastasis (M1). The conventional thinking is that surgical intervention is not indicated for patients with such a metastasis. We have reported the advantage of extensive lymph node dissection, including the neck, for the survival of patients with esophageal carcinoma. ${ }^{4}$ Therefore, in patients with involved cervical lymph nodes, it is thought that dissection of the involved lymph nodes might provide the possibility for radical cure.

In the present retrospective study, we attempted to determine whether and under what circumstances surgical intervention should be recommended for patients with clinically positive cervical nodes for metastasis from thoracic esophageal carcinoma by pretreatment examination.

\section{Patients and methods}

Radical surgery was attempted as primary treatment in 63 patients of a consecutive 121 patients who were diagnosed as having positive cervical node involvement from primary carcinoma of the thoracic esophagus by pretreatment examination at the National Cancer Center Hospital, Tokyo, between 
Table I. The characteristics of patients with clinically positive cervical node involvement from thoracic esophageal carcinoma who underwent an operation as primary treatment $(1987$ to $1995 ; n=63)$

\begin{tabular}{lc}
\hline Variable & $n$ \\
\hline Male/female & $55 / 8$ \\
Age (y, mean \pm SD) & $61.3 \pm 8.2$ \\
Histologic type & \\
$\quad$ Squamous & 60 \\
Adenocarcinoma & 1 \\
$\quad$ Undifferentiated & 2 \\
Tumor location & \\
Upper & 13 \\
Middle & 44 \\
Lower & 6 \\
T categories & \\
T1 & 8 \\
T2 & 6 \\
T3 & 46 \\
T4 & 3 \\
\hline
\end{tabular}

July 1987 and December 1995. All the patients had a histologic examination of endoscopic biopsy specimens, and patients with synchronous or a history of head and neck tumors were excluded. The characteristics of these patients are listed in Table I. The cervical lymph nodes, including the bilateral supraclavicular nodes, the lower half of the deep cervical nodes, and the cervical paraesophageal nodes, were examined for metastasis from the esophageal tumor for pretreatment classification. Diagnosis of positive cervical node involvement in all patients was made by members of the Department of Surgery, computed tomography and ultrasonography. ${ }^{5}$ All of the patients were examined for synchronous head and neck tumors by members of the Department of Head and Neck Surgery.

None of the patients had distant organ metastasis. In 60 patients the tumor was considered not to have infiltrated adjacent organs. Radical surgery was attempted in 3 patients by a combined partial resection of the infiltrated lung. ${ }^{6}$ Six patients who had intramural metastasis received preoperative chemotherapy with cisplatin and 5-fluorouracil for neoadjuvant setting. ${ }^{7}$ Radical surgery was accomplished with esophagectomy through the right thoracotomy and 3-field dissection with dissection of the positive cervical nodes. Cervical nodes were dissected from exactly the same area through a collar incision where they were detected before the operation.

The median follow-up period of censored cases was 44.6 months. In the patients who underwent a radical operation, the initial recurrence site is analyzed. The postoperative survivals were compared according to 5 factors: (1) tumor location, (2) T categories according to the TNM classification, (3) subdivisions of $\mathrm{N}$ categories classified by the number of involved nodes, according to the supplement of the TNM classification, ${ }^{8}(4)$ the existence of abdominal node involve-
Table II. The inital recurrent site in patients underwent an operation without residual tumor (1987 to 1995;

\begin{tabular}{lc}
$n=43)$ & $n$ \\
\hline Site & \\
\hline Local $(\mathrm{n}=7)$ & 3 \\
$\quad$ Bronchial wall & 3 \\
Pleura & 1 \\
$\quad$ Anastomotic site & \\
Lymph node $(\mathrm{n}=19)$ & 11 \\
$\quad$ Cervical & 5 \\
Mediastinal & 2 \\
Abdominal & 1 \\
Axilla & \\
Distant organ $(\mathrm{n}=7)$ & 4 \\
Lung & 1 \\
Liver & 1 \\
Bone & 1 \\
Abdominal wall & \\
\hline
\end{tabular}

ment in addition to cervical node involvement as an index of wide lymphatic spread, and (5) R categories of residual tumor classification according to the TNM classification. The subdivisions of $\mathrm{N}$ categories were classified as N1a (1 to 3 nodes involved), N1b (4 to 7 nodes involved), and N1c (more than 7 nodes involved). The definition of $\mathrm{R}_{0}$ was classified as "no residual tumor." The survival analysis was not performed according to stage, because all the patients had metastasis in cervical lymph node and were classified as stage IV. The patients with a tumor in the upper thoracic esophagus were classified as a mean equivalent to stage IVA, and the patients with tumors in the middle and lower esophagus were classified as a mean equivalent to stage IVB. Thus the analysis performed with attention to the location on the tumor has the same mean as that performed with attention to the stage. The survivals and the survival curves are expressed according to the Kaplan-Meier method and included patients who died of operative complications. The difference between the survival curves was analyzed by the log rank method. The Cox proportional hazards model was used for multivariable analysis of these factors.

\section{Results}

Of 63 patients in whom radical surgery was attempted, unexpected residual tumors were left during surgery in 14 patients, for primary tumor infiltration to adjacent structures in 6 patients, for metastatic node invading adjacent structures in 7 patients, and for unexpected pulmonary metastasis in 1 patient. By histologic examination of dissected nodes, 10 patients had involved nodes only in the neck, and 5 of them had only 1 involved node. Death related to operative complications was observed in 1 patient.

In 49 patients who underwent radical surgery, cancer recurrence was found in 33 patients. The initial recur- 


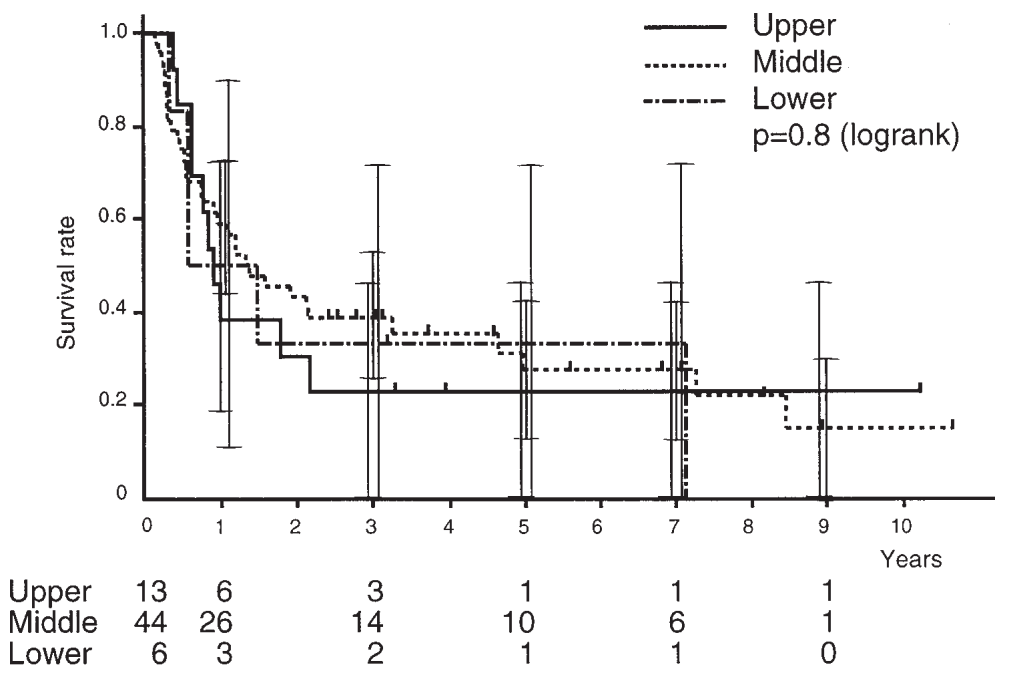

Fig 1. Survival curves expressed according to the Kaplan-Meier method based on tumor location in patients with clinically positive cervical nodes. Vertical bars depict $95 \%$ confidence limits, and the table at the bottom indicates the number of patients at risk at the points.

Table III. The 5-year survivals by the Kaplan-Meier method and the multivariable analysis by the Cox proportional hazards model, compared according to the factors of the patients treated with an operation for thoracic esophageal carcinoma with clinically positive cervical node involvement $(1987-1995 ; n=63)$

\begin{tabular}{|c|c|c|c|c|c|}
\hline & \multirow[b]{2}{*}{$n$} & \multirow[b]{2}{*}{ Five-year survival (\%) } & \multicolumn{3}{|c|}{ Multivariable analysis } \\
\hline & & & $\mathrm{P}$ value & Hazard ratio & 95\% Confidence limits \\
\hline Tumor location & & & .6 & & \\
\hline Upper & 13 & 23.1 & & & \\
\hline Middle & 44 & 27.5 & .8 & 1.084 & $0.414-2.838$ \\
\hline Lower & 6 & 33.3 & .4 & 1.782 & $0.404-7.848$ \\
\hline $\mathrm{T}$ categories & & & .02 & & \\
\hline $\mathrm{T} 1$ & 8 & 87.5 & .005 & 0.032 & $0.003-0.359$ \\
\hline $\mathrm{T} 2$ & 6 & 66.7 & .01 & 0.110 & $0.020-0.599$ \\
\hline $\mathrm{T} 3$ & 46 & 13.7 & .04 & 0.261 & $0.072-0.938$ \\
\hline $\mathrm{T} 4$ & 3 & 0 & & & \\
\hline Subdivisions of $\mathrm{N}$ categories & & & .19 & & \\
\hline N1a (1-3 nodes involved) & 20 & 46.2 & .18 & 0.529 & $0.210-1.334$ \\
\hline N1b (4-7 nodes involved) & 14 & 28.6 & .10 & 0.521 & $0.237-1.145$ \\
\hline N1c (>7 nodes involved) & 29 & 11.5 & & & \\
\hline \multicolumn{6}{|l|}{ Abdominal node involvement } \\
\hline Negative & 30 & 35.6 & .9 & 1.014 & $0.384-2.677$ \\
\hline Positive & 33 & 17.7 & & & \\
\hline \multicolumn{6}{|l|}{ Residual tumor classification } \\
\hline $\mathrm{R}_{0}$ (no residual tumor) & 49 & 34.3 & $<.0001$ & 5.522 & $2.542-11.996$ \\
\hline $\mathrm{R}_{1}, \mathrm{R}_{2}$ (residual tumor) & 14 & 0 & & & \\
\hline
\end{tabular}

rence site is presented in Table II. The initial recurrence was found in the cervical lymph node in 11 patients. After receiving radiotherapy for the recurrence in the cervical node, 3 patients were still alive more than 3 years.

The 5-year survival of the 63 patients who underwent an operation for primary therapy was $26.7 \%$. All 6 patients who received preoperative chemotherapy for intramural metastasis died with recurrence within 24 months. The 5-year survivals of these 63 patients, as compared with the factors listed earlier, are given in Table III. There was no statistically significant difference between the postoperative survival curves when compared according to the location of the tumors (Fig 


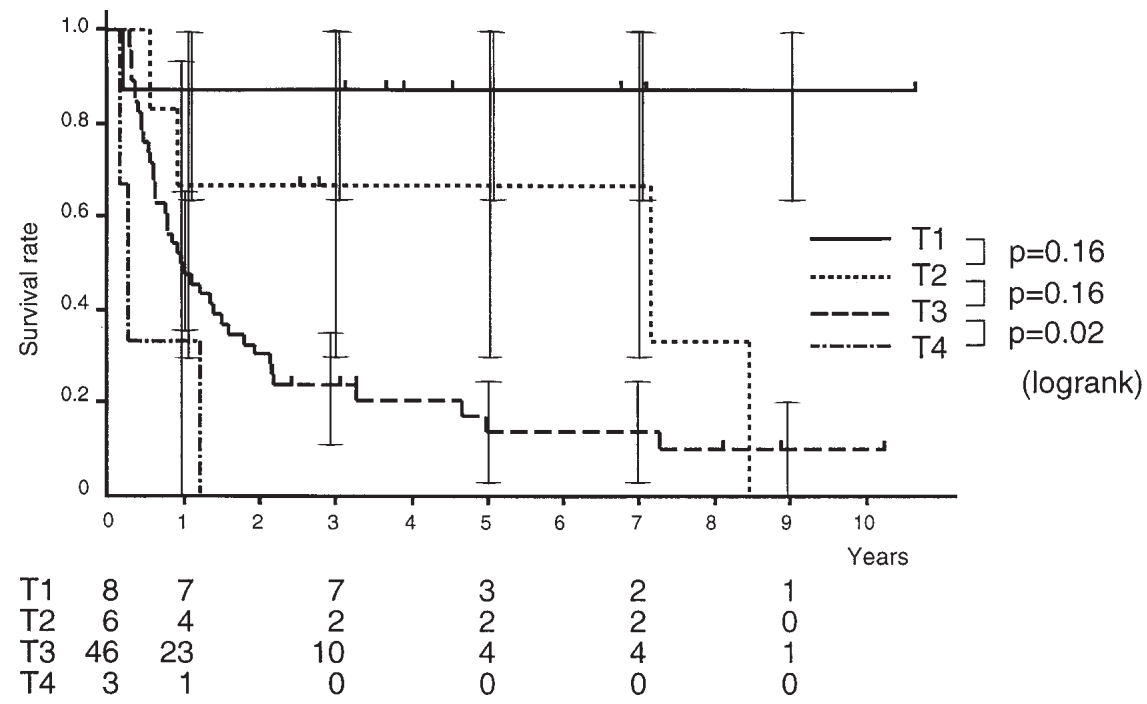

Fig 2. Survival curves expressed according to the Kaplan-Meier method based on $\mathrm{T}$ categories according to the TNM classification in patients with clinically positive cervical nodes. Vertical bars depict $95 \%$ confidence limits, and the table at the bottom indicates the number of patients at risk at the points.

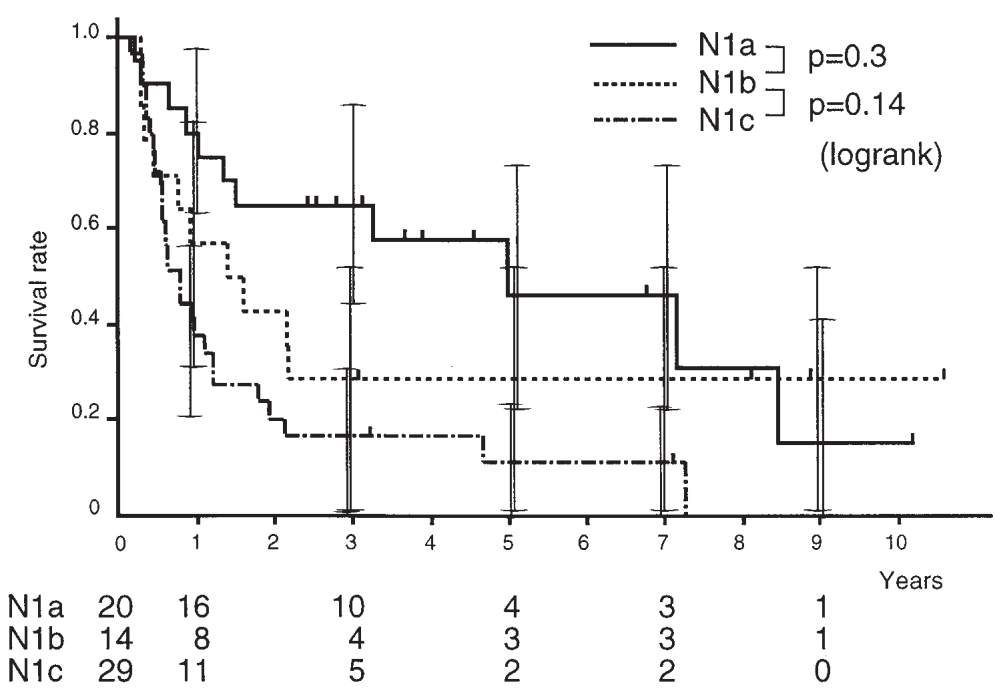

Fig 3. Survival curves expressed according to the Kaplan-Meier method based on subdivisions of N categories classified by the number of involved nodes according to the supplement of the TNM classification in patients with clinically positive cervical nodes (N1a, 1-3 nodes involved; N1b, 4-7 nodes involved; N1c, more than 7 nodes involved). Vertical bars depict $95 \%$ confidence limits, and the table at the bottom indicates the number of patients at risk at the points.

1). The patients with less tumor invasion had a better chance of survival (Fig 2). The patients with tumor invading adjacent structures (T4) had statistically less chance of survival than those without. There was no statistical difference in survival on the basis of the number of involved nodes (Fig 3). The patients without abdominal node involvement had better survivals on the basis of univariate analysis (Fig 4). However, even the patient with the worst factors had a chance of surviving for 5 years after the operation, and it is apparent that those were not important prognostic factors by multivariable analysis (Table III). Analysis revealed the most statistically significant difference between the patients who received resection without residual tumor 


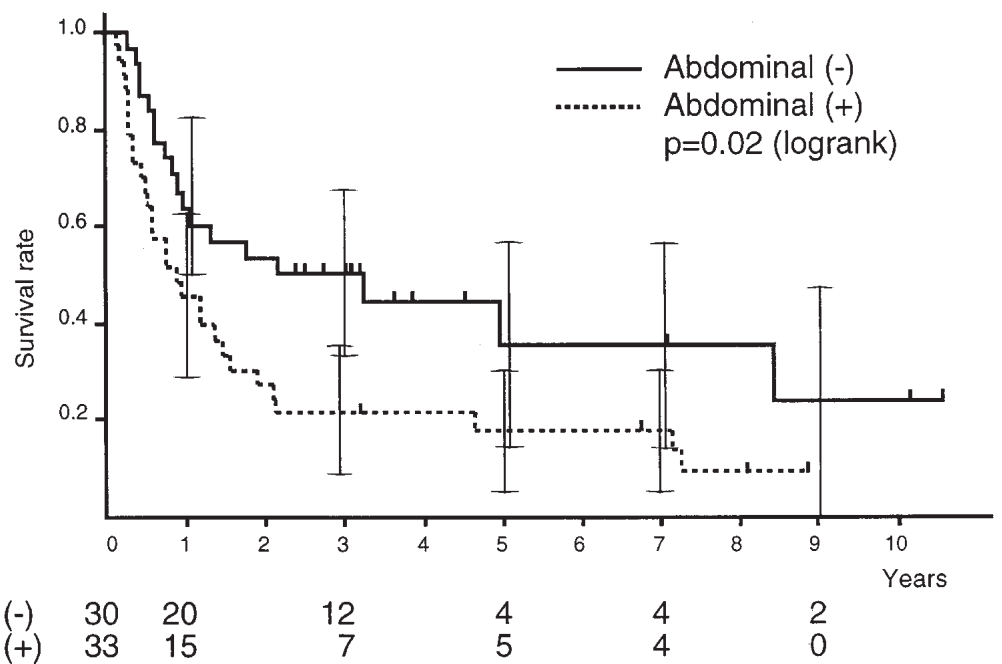

Fig 4. Survival curves expressed according to the Kaplan-Meier method based on the existence of abdominal node involvement as an index of wide lymphatic spread in patients with clinically positive cervical nodes. Vertical bars depict $95 \%$ confidence limits, and the table at the bottom indicates the number of patients at risk at the points.
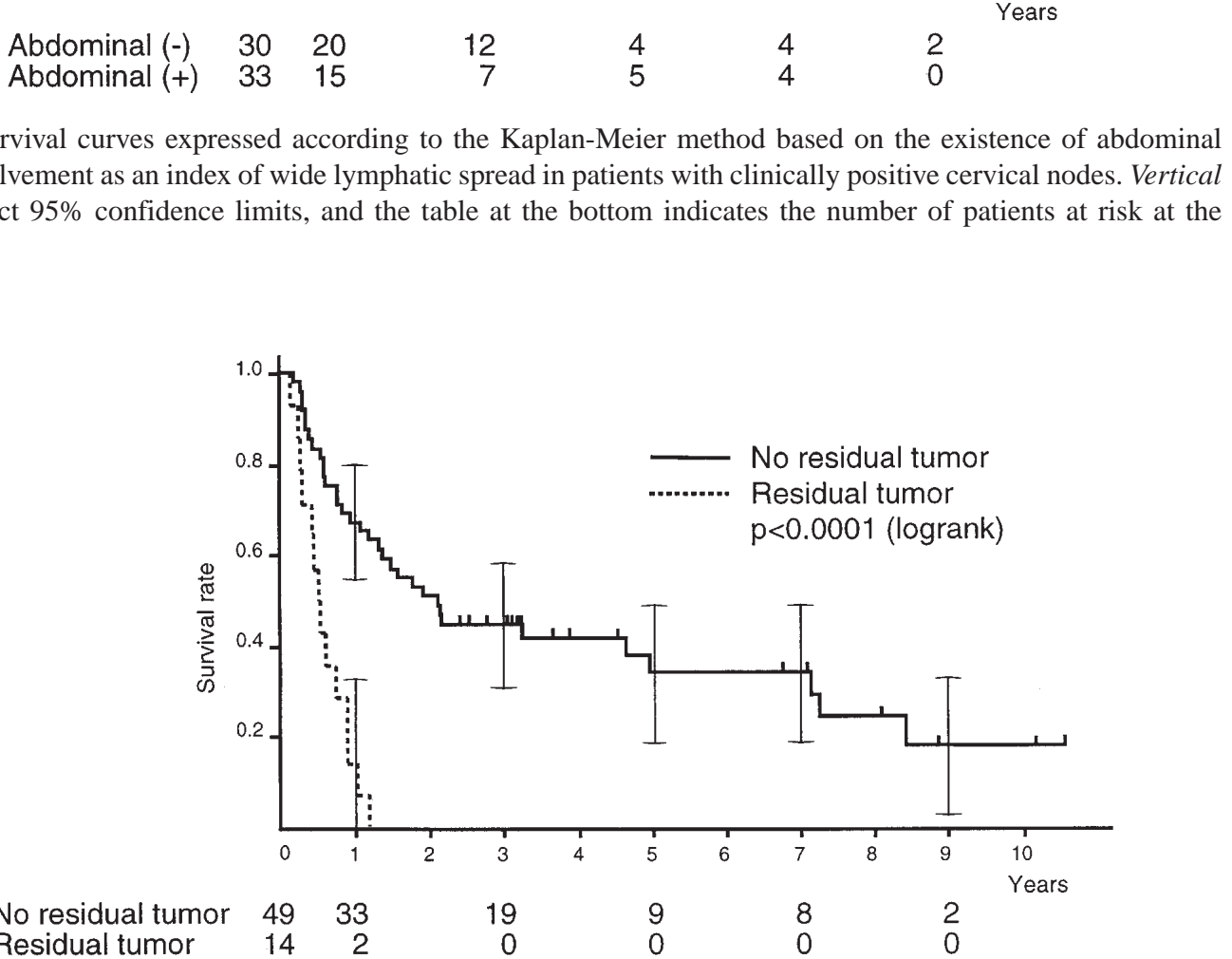

Fig 5. Survival curves expressed according to the Kaplan-Meier method based on R categories of residual tumor classification according to the TNM classification in patients who underwent an operation. A statistically significant difference was observed between survival curves of the patients who received curative resection (no residual tumor) and those who did not (residual tumor). Vertical bars depict 95\% confidence limits, and the table at the bottom indicates the number of patients at risk at the points.

and those who did with residual tumor (Fig 5). Multivariable analysis revealed a significant difference between the patients who received curative resection $\left(\mathrm{R}_{0}\right)$ and those who did not.

\section{Discussion}

The high incidence of cervical lymph node involvement in patients with thoracic esophageal carcinoma is well known. In our series of investigation, 121 patients (19.1\%) of 633 patients with thoracic esophageal carcinoma had clinically positive cervical node involvement by pretreatment examination. Altorki and Skinner ${ }^{2}$ recently reported that cervical nodal metastasis occurred irrespective of tumor location or $\mathrm{T}$ status, even in adenocarcinoma of the esophagus. Also in our series, patients in whom the tumor was in the lower esophagus and the tumor invasion was 
limited to within the submucosal layer (T1) had clinically positive cervical nodal metastasis.

From our experience of extensive lymph node dissection, including the neck, for the patients with esophageal carcinoma, it is thought that dissection of the involved cervical node might provide the possibility for radical cure. The 5-year survival of the 63 patients who underwent an operation was $26.7 \%$. This indicates that some of the patients with cervical involvement were at a stage that was not too late for undergoing an operation. We examined the prognostic factors to determine the indications for surgical intervention for the patients with cervical involvement. In the TNM classification, cervical node involvement was classified as M1a for the patients with a tumor in the upper thoracic esophagus and M1b for those with tumors in the middle and lower esophagus. However, there was no statistically significant difference between the postoperative survival of the patients with cervical involvement when compared according to the location of the tumors.

When survival was compared according to the factors outlined by the TNM classification and the 1993 supplement, patients with the tumor invading more and patients with more involved nodes had worse survivals. As a matter of course, it means that the patients with later stages of the disease had worse survival. The patients with transmural tumors (T3 or T4) had a survival at 5 years of less than $15 \%$. In contrast, there is a dramatic 5-year survival seen in patients with $\mathrm{T} 1$ or $\mathrm{T} 2$ tumors. Similarly, patients with preoperative evidence of extensive lymph node involvement (N1C disease) were given their overall poor 5-year survival. However, multivariable analysis revealed a significant difference in the patients with tumor invading adjacent structures (T4; only 3 of 63 patients). Multivariable analysis revealed a significant difference between the patients who received curative resection $\left(\mathrm{R}_{0}\right)$ and those who did not. Other factors did not exclude the necessity for surgical intervention.

Of 49 patients who underwent curative operations, the initial recurrence was found in the lymph node in 19 patients and in the cervical lymph node in 11 patients. This indicates the difficulty of sugical complete dissection for lymphatic spread. The lymph node spread ends nowhere, and the tumor extends into perinodal fat tissues around the adjacent organs. However, these patients who underwent radical operations had a $34.3 \%$ 5-year survival after the operation. Even when the patients had clinically positive cervical nodes from thoracic esophageal carcinoma, they had a possibility of long-term survival after curative resection with neck lymph node dissection. An operation is thus indicated for curative intent. A radical operation was attempted in 63 patients; however, an operation without residual tumor was accomplished in 49 patients. Accurate pretreatment evaluation is important for deciding which patients require an operation and how to carry out a curative surgical procedure.

We thank Dr. Naohito Yamaguchi, Chief of Cancer Information and Epidemiology Division, National Cancer Center Research Institute, for the statistical consultation.

\section{REFERENCES}

1. Kato H, Tachimori Y, Watanabe H, lizuka T, Terui S, Itabashi M, et al. Lymph node metastasis in thoracic esophageal carcinoma. J Surg Oncol 1991;48:106-11.

2. Altorki NK, Skinner DB. Occult cervical nodal metastasis in esophageal cancer: preliminary results of three-field lymphadenectomy. J Thorac Cardiovasc Surg 1997;113:540-4.

3. Sobin LH, Wittekind Ch, editors. UICC TNM classification on malignant tumors. 5th ed. New York: Wiley-Liss; 1997. p. 54-8.

4. Kato H, Watanabe H, Tachimori Y, Iizuka T. Evaluation of neck lymph node dissection for thoracic esophageal carcinoma. Ann Thorac Surg 1991;51:931-5.

5. Tachimori Y, Karo H, Watanabe H, Yamaguchi H. Neck ultrasonography for thoracic esophageal carcinoma. Ann Thorac Surg 1994:57:1180-3.

6. Kato H, Tachimori Y, Watanabe H, Itabashi M. Surgical treatment of thoracic esophageal carcinoma directly invading the lung. Cancer 1992;70:1457-61.

7. Kato H, Tachimori $\mathrm{Y}$, Watanabe $\mathrm{H}$, Itabashi M, Hirota $\mathrm{H}$, Yamaguchi $\mathrm{H}$, et al. Intramural metastasis of thoracic esophageal carcinoma. Int J Cancer 1992;50:49-52.

8. Hermanek P, Henson DE, Hutter RVP, Sobin LH, editors. UICC TNM supplement 1993. Berlin: Springer-Verlag; 1993. p. 120. 